\title{
On coefficient inequalities in the Carathéodory class of functions
}

\author{
by AdAm LeCKo (Rzeszów)
}

\begin{abstract}
Some inequalities are proved for coefficients of functions in the class $P(\alpha)$, where $\alpha \in[0,1)$, of functions with real part greater than $\alpha$. In particular, new inequalities for coefficients in the Carathéodory class $P(0)$ are given.
\end{abstract}

1. Main results. Let $\mathbb{D}=\{z \in \mathbb{C}:|z|<1\}$ denote the unit disk. We denote by $P(\alpha)$, where $\alpha \in[0,1)$, the class of functions $p$ regular in $\mathbb{D}$ of the form

$$
p(z)=1+\sum_{n=1}^{\infty} p_{n} z^{n}, \quad z \in \mathbb{D},
$$

such that $\operatorname{Re} p(z)>\alpha$ for $z \in \mathbb{D}$ (see [2, p. 105]). The well known class of Carathéodory functions having positive real part in $\mathbb{D}$, denoted by $P$, coincides with $P(0)$. The class $P(\alpha)$, although not explicitly defined, appeared first in [4], where Robertson defined functions convex of order $\alpha$ and starlike of order $\alpha$.

Using the well known estimates $\left|p_{n}\right| \leq 2, n \in \mathbb{N},[1 ; 2$, p. 80] for the coefficients of $p \in P$ it is easy to prove the lemma below (see [2, p. 101]).

Lemma 1.1. Fix $\alpha \in[0,1)$ and let $q$ of the form

$$
q(z)=q_{0}+\sum_{n=1}^{\infty} q_{n} z^{n}, \quad z \in \mathbb{D},
$$

be regular in $\mathbb{D}$. If $\operatorname{Re} q(z)>\alpha$ for $z \in \mathbb{D}$, then

$$
\left|q_{n}\right| \leq 2\left(\operatorname{Re} q_{0}-\alpha\right), \quad n \in \mathbb{N} .
$$

Estimates (1.3) are sharp.

2000 Mathematics Subject Classification: Primary 30C45.

Key words and phrases: Carathéodory class, coefficient inequalities. 
An extremal function for which equalities hold in (1.3) is

$$
q(z)=\frac{q_{0}+\left(\bar{q}_{0}-2 \alpha\right) z}{1-z}, \quad z \in \mathbb{D} .
$$

REMARK 1.2. For $\alpha=0$ we have $\left|q_{n}\right| \leq 2 \operatorname{Re} q_{0}, n \in \mathbb{N}$, for the coefficients $q_{n}$ of a function $q$ such that $\operatorname{Re} q(z)>0$ for $z \in \mathbb{D}$.

An interesting generalization of Remark 1.2 can be found in [3].

As an immediate consequence of (1.3) we have the following estimates for the coefficients of $p \in P(\alpha)$ of the form (1.1) which can be found in $[4$, p. 386]:

$$
\left|p_{n}\right| \leq 2(1-\alpha), \quad n \in \mathbb{N} .
$$

Now we formulate two basic theorems of this paper.

Theorem 1.3. Fix $\alpha \in[0,1)$ and $\xi \in \overline{\mathbb{D}}$. If $p \in P(\alpha)$, then the function

$$
q(z)=q(\xi ; z)=\frac{\xi-\bar{\xi} z[(1-2 \alpha) z+\alpha \xi]}{z}+\frac{(z-\xi)(1-\bar{\xi} z)}{z} p(z),
$$

$z \in \mathbb{D}$, is regular in $\mathbb{D}$ and

$$
\operatorname{Re} q(\xi ; z) \geq \alpha, \quad z \in \mathbb{D} \text {. }
$$

Equality holds in (1.6) only if $|\xi|=1$ and

$$
p(z)=p(\alpha, \xi ; z)=\frac{1+(1-2 \alpha) \bar{\xi} z}{1-\bar{\xi} z}, \quad z \in \mathbb{D} .
$$

P r o of. Observe first that the function (1.5) has a removable singularity at $z=0$ since

$$
q(\xi ; 0)=\lim _{z \rightarrow 0} q(\xi ; z)=1+(1-\alpha)|\xi|^{2}-\xi p_{1} .
$$

Assume first that $p$, and hence $q$, is regular on $\partial \mathbb{D}$. For $z=e^{i \theta}, \theta \in \mathbb{R}$, we have

$$
\begin{aligned}
q\left(\xi ; e^{i \theta}\right)= & 2 i \operatorname{Im}\left(\xi e^{-i \theta}\right)-\alpha|\xi|^{2} \\
& +2 \alpha \bar{\xi} e^{i \theta}+\left[1+|\xi|^{2}-2 \operatorname{Re}\left(\xi e^{-i \theta}\right)\right] p\left(e^{i \theta}\right) .
\end{aligned}
$$

Since $p \in P(\alpha)$ we see that

$$
\begin{aligned}
\operatorname{Re} q\left(\xi ; e^{i \theta}\right)= & -\alpha|\xi|^{2}+2 \alpha \operatorname{Re}\left(\bar{\xi} e^{i \theta}\right) \\
& +\left[\left(1+|\xi|^{2}\right)-2 \operatorname{Re}\left(\xi e^{-i \theta}\right)\right] \operatorname{Re} p\left(e^{i \theta}\right) \\
\geq & \alpha+2 \alpha\left(\operatorname{Re}\left(\bar{\xi} e^{i \theta}\right)-\operatorname{Re}\left(\xi e^{-i \theta}\right)\right)=\alpha .
\end{aligned}
$$

By the mimimum principle for harmonic functions the above inequality holds in $\overline{\mathbb{D}}$, i.e. $\operatorname{Re} q(\xi ; z) \geq \alpha$ for $z \in \overline{\mathbb{D}}$.

If $p$ is not regular on $\partial \mathbb{D}$, then we consider the functions $p_{r}(z)=p(r z)$, $z \in \mathbb{D}$, for $r \in(0,1)$. Replacing $p$ by $p_{r}$ on the right hand side of $(1.5)$ we obtain the corresponding function $q_{r}(\xi ; z), z \in \mathbb{D}$. Repeating the above 
considerations we get the strict inequality $\operatorname{Re} q_{r}(\xi ; z)>\alpha$ for $z \in \mathbb{D}$. Letting $r \rightarrow 1$ we see that $p_{r} \rightarrow p$ and $q_{r} \rightarrow q$. Consequently, $\operatorname{Re} q(\xi ; z) \geq \alpha$ for $z \in \mathbb{D}$.

If $|\xi|<1$, then from $(1.5)$ we have $q(\xi ; \xi)=1-(1-\alpha)|\xi|^{2}>\alpha$. Hence $\operatorname{Re} q(\xi ; z)>\alpha$ for $z \in \mathbb{D}$ and $|\xi|<1$.

If $|\xi|=1$, then from (1.5) we deduce that

$$
\begin{aligned}
\operatorname{Re} q(\xi ; 0)-\alpha & =(1-\alpha)\left(1+|\xi|^{2}\right)-\operatorname{Re}\left(\xi p_{1}\right) \\
& \geq(1-\alpha)\left(1+|\xi|^{2}\right)-\left|\xi p_{1}\right| \geq(1-\alpha)(1-|\xi|)^{2}=0
\end{aligned}
$$

for all $\alpha \in[0,1)$. Equality holds only if $\operatorname{Re}\left(\xi p_{1}\right)=\left|\xi p_{1}\right|$ and $\left|p_{1}\right|=2(1-\alpha)$. Hence $p_{1}=2(1-\alpha) \bar{\xi}$, which holds only for the function $p$ defined by (1.7). Then $q(\xi ; z)=\alpha$ for each $\xi \in \partial \mathbb{D}$ and all $z \in \mathbb{D}$.

Now we prove the converse theorem for $\xi \in \mathbb{D}$.

Theorem 1.4. Fix $\alpha \in[0,1)$ and $\xi \in \mathbb{D}$. Assume that $q$ is regular in $\mathbb{D}$, $\operatorname{Re} q(z)>\alpha$ for $z \in \mathbb{D}$ and

$$
q(\xi)=1-(1-\alpha)|\xi|^{2} .
$$

Then the function

$$
\begin{aligned}
p(z) & =p(\xi ; z) \\
& =\frac{z}{(z-\xi)(1-\bar{\xi} z)}\left(q(z)-\frac{\xi-\bar{\xi} z[(1-2 \alpha) z+\alpha \xi]}{z}\right), \quad z \in \mathbb{D},
\end{aligned}
$$

is regular in $\mathbb{D}$ and $p \in P(\alpha)$.

Pr o of. Simple calculations lead to

$$
p(\xi ; \xi)=\lim _{z \rightarrow \xi} p(\xi ; z)=\frac{1+(1-2 \alpha)|\xi|^{2}+\xi q^{\prime}(\xi)}{1-|\xi|^{2}}
$$

so at $z=\xi$ the function $p$ has a removable singularity. Moreover $p(\xi ; 0)=1$ for each $\xi \in \mathbb{D}$. Therefore $p$ is regular in $\mathbb{D}$ and of the form (1.1) for each $\xi \in \mathbb{D}$.

Now we prove that $p \in P(\alpha)$. Assume first that $q$ is regular on $\partial \mathbb{D}$. By (1.10), so is $p$, and for $z=e^{i \theta}, \theta \in \mathbb{R}$, we have

$$
p\left(\xi ; e^{i \theta}\right)=\frac{1}{1+|\xi|^{2}-2 \operatorname{Re}\left(\xi e^{-i \theta}\right)}\left(q\left(e^{i \theta}\right)+\alpha|\xi|^{2}-2 \alpha \bar{\xi} e^{i \theta}-2 i \operatorname{Im}\left(\bar{\xi} e^{i \theta}\right)\right) .
$$

Since $\operatorname{Re} q(z) \geq \alpha$ for $z \in \partial \mathbb{D}$ we obtain

$$
\begin{aligned}
\operatorname{Re} p\left(\xi ; e^{i \theta}\right) & =\frac{1}{1+|\xi|^{2}-2 \operatorname{Re}\left(\xi e^{-i \theta}\right)}\left(\operatorname{Re} q\left(e^{i \theta}\right)+\alpha|\xi|^{2}-2 \alpha \operatorname{Re}\left(\bar{\xi} e^{i \theta}\right)\right) \\
& \geq \frac{1+|\xi|^{2}-2 \operatorname{Re}\left(\bar{\xi} e^{i \theta}\right)}{1+|\xi|^{2}-2 \operatorname{Re}\left(\xi e^{-i \theta}\right)} \alpha=\alpha .
\end{aligned}
$$


By the mimimum principle for harmonic functions the above inequality is true in $\overline{\mathbb{D}}$, i.e. $\operatorname{Re} p(\xi ; z) \geq \alpha$ for $z \in \overline{\mathbb{D}}$.

If $q$ is not regular on $\partial \mathbb{D}$, then arguing as in the part of Theorem 1.3 concerning $p_{r}$ (setting $q_{r}(z)=q(r z), z \in \mathbb{D}$, for $r \in(0,1)$, and using (1.10) in place of $(1.5))$ we obtain $\operatorname{Re} p(\xi ; z) \geq \alpha$ for $z \in \mathbb{D}$.

Finally, recall that $p(\xi ; 0)=1$ for each $\xi \in \mathbb{D}$. This implies that $\operatorname{Re} p(\xi ; z)$ $>\alpha$ for $z \in \mathbb{D}$ and $\xi \in \mathbb{D}$. Therefore $p \in P(\alpha)$.

For $\alpha=0$ we obtain from Theorems 1.3 and 1.4 the following results.

Corollary 1.5. Fix $\xi \in \overline{\mathbb{D}}$. If $p \in P$, then the function

$$
q(z)=q(\xi ; z)=\frac{\xi-\bar{\xi} z^{2}}{z}+\frac{(z-\xi)(1-\bar{\xi} z)}{z} p(z), \quad z \in \mathbb{D},
$$

is regular in $\mathbb{D}$ and $\operatorname{Re} q(\xi ; z) \geq 0$ for $z \in \mathbb{D}$. Equality holds only if $|\xi|=1$ and

$$
p(z)=p(0, \xi ; z)=\frac{1+\bar{\xi} z}{1-\bar{\xi} z}, \quad z \in \mathbb{D} .
$$

Corollary 1.6. Fix $\xi \in \mathbb{D}$. Assume that $q$ is regular in $\mathbb{D}, \operatorname{Re} q(z)>0$ for $z \in \mathbb{D}$ and $q(\xi)=1-|\xi|^{2}$. Then the function

$$
p(z)=p(\xi ; z)=\frac{z}{(z-\xi)(1-\bar{\xi} z)}\left(q(z)-\frac{\xi-\bar{\xi} z^{2}}{z}\right), \quad z \in \mathbb{D},
$$

is regular in $\mathbb{D}$ and $p \in P$.

For $\xi=1$ Corollary 1.5 is due to Robertson [5].

2. Applications. In this section we apply Theorem 1.3 to obtain some inequalities for coefficients of functions in the class $P(\alpha)$. In the case when $\alpha=0$ these results generalize the well known estimates for coefficients of functions in the Carathéodory class.

Theorem 2.1. Fix $\alpha \in[0,1)$ and $\xi \in \overline{\mathbb{D}}$. If $p \in P(\alpha)$ and $p$ is of the form (1.1), then

$$
\begin{aligned}
& \left|\xi p_{2}-\left(1+|\xi|^{2}\right) p_{1}+2(1-\alpha) \bar{\xi}\right| \leq 2\left((1-\alpha)\left(1+|\xi|^{2}\right)-\operatorname{Re}\left(\xi p_{1}\right)\right), \\
& \left|\xi p_{n+1}-\left(1+|\xi|^{2}\right) p_{n}+\bar{\xi} p_{n-1}\right| \leq 2\left((1-\alpha)\left(1+|\xi|^{2}\right)-\operatorname{Re}\left(\xi p_{1}\right)\right) \\
& |||\xi| p_{n+1}-p_{n}|-| \xi|\cdot||\xi| p_{n}-p_{n-1}|| \leq 2\left((1-\alpha)\left(1+|\xi|^{2}\right)-|\xi| \operatorname{Re} p_{1}\right)
\end{aligned}
$$

for $n=2,3, \ldots$ Estimates (2.1)-(2.3) are sharp.

Proof. By Theorem 1.3 the function $q$ defined by (1.5) is regular in $\mathbb{D}$ and $\operatorname{Re} q(\xi ; z) \geq \alpha$ for $z \in \mathbb{D}$. We can assume that $q$ is of the form (1.2). 
From (1.5) we have

$$
\begin{aligned}
z q(\xi ; z)= & {\left[1+(1-\alpha)|\xi|^{2}-\xi p_{1}\right] z+\left[-\xi p_{2}+\left(1+|\xi|^{2}\right) p_{1}-2(1-\alpha) \bar{\xi}\right] z^{2}+\ldots } \\
& +\left[-\xi p_{n}+\left(1+|\xi|^{2}\right) p_{n-1}-\bar{\xi} p_{n-2}\right] z^{n}+\ldots
\end{aligned}
$$

Consequently,

$$
q_{0}=1+(1-\alpha)|\xi|^{2}-\xi p_{1}, \quad q_{1}=-\xi p_{2}+\left(1+|\xi|^{2}\right) p_{1}-2(1-\alpha) \bar{\xi}
$$

and

$$
q_{n}=-\xi p_{n}+\left(1+|\xi|^{2}\right) p_{n-1}-\bar{\xi} p_{n-2} \quad \text { for } n=2,3, \ldots
$$

Now using (1.3) and the formula for $q_{0}$ we obtain (2.1) and (2.2).

To prove (2.3) assume that $\xi=|\xi| e^{i \varphi}, \varphi \in[0,2 \pi)$. Since $p \in P(\alpha)$, the function $p\left(e^{-i \varphi} z\right), z \in \mathbb{D}$, also belongs to $P(\alpha)$, and applying $(2.2)$ to it we have

$$
\begin{aligned}
|||\xi| p_{n+1}-p_{n}|-| \xi|\cdot| & || \xi\left|p_{n}-p_{n-1}\right| \mid \\
& =|| \xi e^{-i \varphi} p_{n+1}-p_{n}|-| \bar{\xi}|\cdot| \xi e^{-i \varphi} p_{n}-p_{n-1}|| \\
& \leq\left|\xi e^{-i \varphi} p_{n+1}-\left(1+|\xi|^{2}\right) p_{n}+\bar{\xi} e^{i \varphi} p_{n-1}\right| \\
& \leq 2\left((1-\alpha)\left(1+|\xi|^{2}\right)-\operatorname{Re}\left(\xi e^{-i \varphi} p_{1}\right)\right) \\
& =2\left((1-\alpha)\left(1+|\xi|^{2}\right)-|\xi| \operatorname{Re} p_{1}\right) .
\end{aligned}
$$

The function

$$
p(\alpha, 1 ; z)=\frac{1+(1-2 \alpha) z}{1-z}=1+2(1-\alpha) \sum_{n=1}^{\infty} z^{n}, \quad z \in \mathbb{D},
$$

is in $P(\alpha)$ and gives equalities in (2.1)-(2.3).

For $\alpha=0$ the above yields the following result.

Corollary 2.2. Fix $\xi \in \overline{\mathbb{D}}$. If $p \in P$ and $p$ is of the form (1.1), then

$$
\begin{aligned}
& \left|\xi p_{2}-\left(1+|\xi|^{2}\right) p_{1}+2 \bar{\xi}\right| \leq 2\left(1+|\xi|^{2}-\operatorname{Re}\left(\xi p_{1}\right)\right) \\
& \left|\xi p_{n+1}-\left(1+|\xi|^{2}\right) p_{n}+\bar{\xi} p_{n-1}\right| \leq 2\left(1+|\xi|^{2}-\operatorname{Re}\left(\xi p_{1}\right)\right), \\
& |||\xi| p_{n+1}-p_{n}|-| \xi|\cdot||\xi| p_{n}-p_{n-1}|| \leq 2\left(1+|\xi|^{2}-|\xi| \operatorname{Re} p_{1}\right)
\end{aligned}
$$

for $n=2,3, \ldots$ Estimates (2.5)-(2.7) are sharp.

Corollary 2.3. If $p \in P$ and $p$ is of the form (1.1), then

$$
\begin{aligned}
& \left|p_{2}-2 p_{1}+2\right| \leq 2 \operatorname{Re}\left(2-p_{1}\right), \\
& \left|p_{n+1}-2 p_{n}+p_{n-1}\right| \leq 2\left(2-\operatorname{Re} p_{1}\right), \\
& \left|p_{2}+2 p_{1}+2\right| \leq 2\left(2+\operatorname{Re} p_{1}\right), \\
& \left|p_{n+1}+2 p_{n}+p_{n-1}\right| \leq 2\left(2+\operatorname{Re} p_{1}\right),
\end{aligned}
$$




$$
\begin{aligned}
& \left|p_{2}+2 i p_{1}-2\right| \leq 2\left(2+\operatorname{Im} p_{1}\right) \\
& \left|p_{n+1}+2 i p_{n}-p_{n-1}\right| \leq 2\left(2+\operatorname{Im} p_{1}\right), \\
& \left|p_{2}-2 i p_{1}-2\right| \leq 2\left(2-\operatorname{Im} p_{1}\right) \\
& \left|p_{n+1}-2 i p_{n}-p_{n-1}\right| \leq 2\left(2-\operatorname{Im} p_{1}\right)
\end{aligned}
$$

for $n=2,3, \ldots$ All estimates are sharp.

Proof. Estimates (2.8) follow from (2.5) and (2.6) by setting $\xi=1$. Setting $\xi=-1$ in (2.5) and (2.6) we obtain (2.9). Analogously, setting $\xi=i$ and $\xi=-i$ in (2.5) and (2.6) we get (2.10) and (2.11), respectively. The function $p(z)=p(0, \xi ; z), z \in \mathbb{D}$, defined by (1.6), for suitable $\xi$ as above, is extremal for the cases considered.

Taking $|\xi|=1$ in (2.7) we have

Corollary 2.4. If $p \in P$ and $p$ is of the form (1.1), then

$$
|| p_{n+1}-p_{n}|-| p_{n}-p_{n-1}|| \leq 2\left(2-\operatorname{Re} p_{1}\right)
$$

for $n=2,3, \ldots$ The estimates are sharp.

Setting $\xi=1 / n$ and $\xi=1-1 / n, n=2,3, \ldots$, in (2.6) we have respectively:

Corollary 2.5. If $p \in P$ and $p$ is of the form (1.1), then

$$
\left|p_{n+1}-\left(n+\frac{1}{n}\right) p_{n}+p_{n-1}\right| \leq 2\left(n+\frac{1}{n}-\operatorname{Re} p_{1}\right)
$$

for $n=2,3, \ldots$ The estimates are sharp.

Corollary 2.6. If $p \in P$ and $p$ is of the form (1.1), then

$$
\left|p_{n+1}-\left(\frac{n}{n-1}+\frac{n-1}{n}\right) p_{n}+p_{n-1}\right| \leq 2\left(\frac{n}{n-1}+\frac{n-1}{n}-\operatorname{Re} p_{1}\right)
$$

for $n=2,3, \ldots$ The estimates are sharp.

Theorem 2.7. Fix $\alpha \in[0,1)$ and $\xi \in \overline{\mathbb{D}}$. If $p \in P(\alpha)$ and $p$ is of the form (1.1), then

$$
\begin{aligned}
& \text { (2.12) }\left|\xi p_{n+1}-p_{n}\right| \\
& \leq \begin{cases}2 \frac{1-|\xi|^{n}}{1-|\xi|}\left[(1-\alpha)\left(1+|\xi|^{2}\right)-\operatorname{Re}\left(\xi p_{1}\right)\right]+\left|2(1-\alpha)-\xi p_{1}\right| \cdot|\xi|^{n}, & |\xi|<1, \\
(2 n+1)\left|2(1-\alpha)-\xi p_{1}\right|, & |\xi|=1,\end{cases}
\end{aligned}
$$


(2.13) $\quad|| \xi p_{n+1}|-| p_{n}||$

$\leq \begin{cases}2 \frac{1-|\xi|^{n}}{1-|\xi|}\left[(1-\alpha)\left(1+|\xi|^{2}\right)-\left|p_{1}\right| \operatorname{Re} \xi\right]+|2(1-\alpha)-\xi| p_{1}|| \cdot|\xi|^{n}, & |\xi|<1, \\ (2 n+1)|2(1-\alpha)-\xi| p_{1}||, & |\xi|=1,\end{cases}$ for $n=2,3, \ldots$ The estimates are sharp for each $\xi \in[0,1]$.

Proof. By Theorem 1.3 the function $q$ defined by (1.5) is regular in $\mathbb{D}$ and $\operatorname{Re} q(\xi ; z) \geq \alpha$ for $z \in \mathbb{D}$. We can assume that $q$ is of the form (1.2).

From (1.5) we have

$$
\begin{aligned}
\frac{q(z)}{1-\bar{\xi} z}= & \frac{\xi-\bar{\xi} z[(1-2 \alpha) z+\alpha \xi]}{z(1-\bar{\xi} z)}+\frac{z-\xi}{z} p(z) \\
= & 1-2 \alpha+\frac{\xi}{z}-\left[(1-\alpha)\left(1-|\xi|^{2}\right)-\alpha\right] \frac{1}{1-\bar{\xi} z}+\left(1-\frac{\xi}{z}\right) p(z) \\
= & {\left[1+(1-\alpha)|\xi|^{2}-\xi p_{1}\right] } \\
& +\left[p_{1}-\xi p_{2}-\left((1-\alpha)\left(1-|\xi|^{2}\right)-\alpha\right) \bar{\xi}\right] z+\ldots+ \\
& +\left[p_{n}-\xi p_{n+1}-\left((1-\alpha)\left(1-|\xi|^{2}\right)-\alpha\right) \bar{\xi}^{n}\right] z^{n}+\ldots
\end{aligned}
$$

But

$$
\begin{aligned}
\frac{q(z)}{1-\bar{\xi} z}= & q_{0}+\left[q_{1}+q_{0} \bar{\xi}\right] z+\ldots+ \\
& +\left[q_{n}+q_{n-1} \bar{\xi}+\ldots+q_{1} \bar{\xi}^{n-1}+q_{0} \bar{\xi}^{n}\right] z^{n}+\ldots
\end{aligned}
$$

By the above and from (2.14) we have

$$
\begin{gathered}
q_{0}=1+(1-\alpha)|\xi|^{2}-\xi p_{1}, \\
q_{1}+q_{0} \bar{\xi}=p_{1}-\xi p_{2}-\left[(1-\alpha)\left(1-|\xi|^{2}\right)-\alpha\right] \bar{\xi}
\end{gathered}
$$

and

$$
q_{n}+q_{n-1} \bar{\xi}+\ldots+q_{1} \bar{\xi}^{n-1}+q_{0} \bar{\xi}^{n}=p_{n}-\xi p_{n+1}-\left[(1-\alpha)\left(1-|\xi|^{2}\right)-\alpha\right] \bar{\xi}^{n}
$$

for all $n \in \mathbb{N}$. From estimates (1.3) it follows that

$$
\begin{aligned}
& \left|\xi p_{n+1}-p_{n}\right| \\
& \quad \leq\left|q_{n}+q_{n-1} \bar{\xi}+\ldots+q_{1} \bar{\xi}^{n-1}\right|+\left|q_{0}+(1-\alpha)\left(1-|\xi|^{2}\right)-\alpha\right| \cdot|\xi|^{n} \\
& \quad \leq 2\left(\operatorname{Re} q_{0}-\alpha\right)\left(1+|\xi|+\ldots+|\xi|^{n-1}\right)+\left|2(1-\alpha)-\xi p_{1}\right| \cdot|\xi|^{n}
\end{aligned}
$$

which gives estimates (2.12).

In order to prove (2.13) assume that $p_{1}=\left|p_{1}\right| e^{i \psi}, \psi \in[0,2 \pi)$. Let $|\xi|=1$. Since $p \in P(\alpha)$, the function $p\left(e^{-i \psi} z\right), z \in \mathbb{D}$, also belongs to $P(\alpha)$, and applying (2.12) we have

$$
\begin{aligned}
|| \xi p_{n+1}|-| p_{n}|| & \leq\left|\xi e^{-i \psi} p_{n+1}-p_{n}\right| \leq(2 n+1)\left|2(1-\alpha)-\xi e^{-i \psi} p_{1}\right| \\
& =|2(1-\alpha)-\xi| p_{1}|| .
\end{aligned}
$$


Analogously we prove $(2.13)$ when $|\xi|<1$.

If $\xi \in[0,1)$, then equalities in (2.12) and (2.13) are achieved for the coefficients of the function (2.4).

For $\xi=1$ the factor $2 n+1$ which appears on the right hand side of (2.12) and (2.13) cannot be replaced by a smaller one. To see this consider for each $\alpha \in[0,1)$ and $\theta \in[0,2 \pi)$ the function

$$
\begin{aligned}
p_{\alpha, \theta}(z) & =\frac{1-2 \alpha z \cos \theta-(1-2 \alpha) z^{2}}{1-2 z \cos \theta+z^{2}} \\
& =1+2(1-\alpha) \sum_{n=2}^{\infty} \cos (n \theta) z^{n}, \quad z \in \mathbb{D} .
\end{aligned}
$$

Then for $\xi=1$ we have

$$
\begin{aligned}
\left|p_{n+1}-p_{n}\right| & =2(1-\alpha)|\cos ((n+1) \theta)-\cos (n \theta)| \\
& =4(1-\alpha)\left|\frac{\sin ((2 n+1) \theta / 2)}{\sin (\theta / 2)}\right| \sin ^{2}(\theta / 2) \\
& \leq 4(1-\alpha)(2 n+1) \sin ^{2}(\theta / 2)
\end{aligned}
$$

for all $\theta \in[0,2 \pi)$. Taking $\theta$ sufficiently small we see that the factor $2 n+1$ is the best possible.

For $\alpha=0$ Theorem 2.7 has the following form.

Corollary 2.8. Fix $\xi \in \overline{\mathbb{D}}$. If $p \in P$ and $p$ is of the form (1.1), then

$$
\begin{aligned}
& \quad\left|\xi p_{n+1}-p_{n}\right| \\
& \quad \leq \begin{cases}2 \frac{1-|\xi|^{n}}{1-|\xi|}\left[1+|\xi|^{2}-\operatorname{Re}\left(\xi p_{1}\right)\right]+\left|2-\xi p_{1}\right| \cdot|\xi|^{n} & \text { for }|\xi|<1, \\
(2 n+1)\left|2-\xi p_{1}\right| & \text { for }|\xi|=1,\end{cases} \\
& \quad|| \xi p_{n+1}|-| p_{n}|| \\
& \leq \begin{cases}2 \frac{1-|\xi|^{n}}{1-|\xi|}\left[1+|\xi|^{2}-\left|p_{1}\right| \operatorname{Re} \xi\right]+|2-\xi| p_{1}|| \cdot|\xi|^{n} & \text { for }|\xi|<1, \\
(2 n+1)|2-\xi| p_{1}|| & \text { for }|\xi|=1,\end{cases}
\end{aligned}
$$

for $n=2,3, \ldots$ The estimates are sharp for $\xi \in[0,1]$.

For $\xi=1$ the result of Corollary 2.8 was obtained by Robertson [5].

Setting $\xi=1 / n, n=2,3, \ldots$, we get from Corollary 2.8 the following results.

Corollary 2.9. If $p \in P$ and $p$ is of the form (1.1), then

$$
\left|p_{n+1}-n p_{n}\right| \leq 2 \frac{1-(1 / n)^{n}}{n-1}\left(n^{2}+1-n \operatorname{Re} p_{1}\right)+\left(\frac{1}{n}\right)^{n}\left|2 n-p_{1}\right|
$$

for $n=2,3, \ldots$ The estimates are sharp. 
In particular, for $n=2$ and $n=3$ we have

$$
\begin{aligned}
& \left|p_{3}-2 p_{2}\right| \leq \frac{15}{2}-3 \operatorname{Re} p_{1}+\left|1-\frac{p_{1}}{4}\right|, \\
& \left|p_{4}-3 p_{3}\right| \leq \frac{1}{27}\left(260-78 \operatorname{Re} p_{1}+\left|6-p_{1}\right|\right) .
\end{aligned}
$$

For $\xi=1-1 /(n+1), n \in \mathbb{N}$, Corollary 2.8 yields

Corollary 2.10. If $p \in P$ and $p$ is of the form (1.1), then

$$
\begin{aligned}
\left|n p_{n+1}-(n+1) p_{n}\right| \leq & 2\left(1-\left(\frac{n}{n+1}\right)^{n}\right)\left(2 n^{2}+2 n+1-n(n+1) \operatorname{Re} p_{1}\right) \\
& +\left(\frac{n}{n+1}\right)^{n}\left|2(n+1)-n p_{1}\right|
\end{aligned}
$$

for $n \in \mathbb{N}$. The estimates are sharp.

In particular, for $n=1$ and $n=2$ we have

$$
\begin{aligned}
\left|p_{2}-2 p_{1}\right| & \leq 5-2 \operatorname{Re} p_{1}+\left|2-\frac{p_{1}}{2}\right|, \\
\left|2 p_{3}-3 p_{2}\right| & \leq \frac{1}{27}\left(260-78 \operatorname{Re} p_{1}+\left|6-p_{1}\right|\right) .
\end{aligned}
$$

\section{References}

[1] C. Carathéodory, Über den Variabilitätsbereich der Koeffizienten von Potenzreihen, die gegebene Werte nicht annehmen, Math. Ann. 64 (1907), 95-115.

[2] A. W. Goodman, Univalent Functions, Mariner, Tampa, FL 1983.

[3] Z. J. Jakubowski and J. Stankiewicz, On some classes of functions with the special normalizations, Folia Sci. Univ. Tech. Res. 73 (1990), 29-48.

[4] M. S. Robertson, On the theory of univalent functions, Ann. of Math. 37 (1936), $374-408$

[5] - Univalent functions starlike with respect to a boundary point, J. Math. Anal. Appl. 81 (1981), 327-345.

Department of Mathematics

Technical University of Rzeszów

W. Pola 2

35-959 Rzeszów, Poland

E-mail: alecko@prz.rzeszow.pl 